Preprint. Final version appears as:

Morgan, R.M., French, J., O’Donnell, L. \& Bull, P.A. (2010) The reincorporation and redistribution of trace geoforensic particulates on clothing: an introductory study. Science and Justice. DOI 10.1016/j.scijus.2010.04.002

The reincorporation and redistribution of trace geoforensic particulates on clothing: An introductory study.

*Morgan, R. M. ${ }^{1}$, French, J. ${ }^{1,2}$, O’Donnell, L. ${ }^{2}$, Bull, P. A. ${ }^{2}$

${ }^{1}$ UCL Department of Security and Crime Science, 35 Tavistock Square, London, WC1H 9EZ

${ }^{2}$ University of Oxford, OUCE, South Parks Road, Oxford, OX1 3QY

Communicating author: Ruth Morgan

ruth.morgan@ucl.ac.uk

Tel: +442031083037

Fax: +442031083088 


\title{
The reincorporation and redistribution of trace geoforensic particulates on clothing:
}

\author{
An introductory study.
}

\begin{abstract}
Two experimental studies were undertaken to investigate the processes of reincorporation and redistribution of trace evidence on garments when worn by a suspect or a victim (reincorporation) or after the garments have been seized and packaged for subsequent forensic analysis (redistribution). The first experiment utilised UV powder, an established proxy for geoforensic trace particulates and the second experiment utilised daffodil pollen transferred onto garments under conditions that mimicked forensic reality. It was demonstrated that reincorporation of trace particulates occurs from upper to lower parts of the same garment and also from upper garments to lower garments. Reincorporation also occurred to all areas of the lower garments, however the highest concentration of particulates was found to be the lap area of the jeans. Particulates also tended to be preserved around technical details such as stitching or relief design features of the garments. Thus the decay of particulates after a contact has been made does not necessarily involve a loss of those particulates from the entire system. These findings have implications for the interpretation of trace evidence when seeking to establish the source of initial contacts or the chronology of pertinent events. The second study demonstrated that folding and packaging items of clothing elicits a redistribution of any trace particulate evidence that is present thereby eliciting an alteration in the spatial distribution of that evidence. There is therefore a necessity to take the context of trace evidence into account and also to follow protocols that are sensitive to these aspects of trace evidence behaviour as a failure to do so may have the consequence of the interpretation of such evidence being at best confused and at worst meaningless.
\end{abstract}

Keywords: trace evidence, UV powder, geoforensic analysis, pollen, clothing, spatial and temporal distribution, forensic protocol. 


\section{Introduction}

It is well established that microscopic trace evidence can often be found on articles of clothing during the course of forensic investigations [1,2] and the transfer and persistence of this evidence has been demonstrated in experimental situations [3-7] in addition to forensic case work [8]. The common finding of these studies has been the decay curve of particulates whereby there is a rapid initial loss of weakly bonded particulates followed by a slower loss of the more strongly bonded particulates [9].

When considering the persistence of trace evidence over time there is an added 'complication' of distinguishing between pre-, syn- and post-forensic event transfers of particulates onto a pertinent item [10-12]. This issue leads to further complications that may arise from transfers of particulates onto garments from particular sources where direct contact with the original source of the particulates has not been made by the garment but is rather, a result of secondary transfers effected by an intermediary. Such scenarios and are an extension of the work of Allen et al. [13] on glass particulates.

The decay of particulates from an item of clothing will not necessarily mean that the particulate is lost from the 'system'. It has been suggested that particulates shed from upper garments may be reincorporated onto lower parts of that garment or indeed other garments on the lower parts of the body (for example from a jumper to a pair of jeans) [7]. It has also been shown that fibres may be further redistributed on a garment during the packaging and transit process [14]. This study examines these two aspects of reincorporation and redistribution which may be two key processes operating on garments when they are being worn by a suspect or victim (reincorporation) or after the garments have been seized and packaged for subsequent forensic analysis (redistribution). There may be considerable implications for the movement, packaging and storing of evidence in forensic enquiry.

In the first instance an experimental study was designed to examine the degree to which the reincorporation of geoforensic particulates on garments occurs. The main questions posed were:

- Do particulates redistribute and reincorporate from higher to lower levels on clothing?

- If so, what is the spatial extent of the reincorporation and redistribution?

In the second instance a further experiment was designed to establish the degree to which geoforensic trace evidence is redistributed on a single garment during the folding and packaging process undertaken prior to forensic examination. The main question addressed here was:

- $\quad$ Are particulates redistributed on a garment during the packaging and subsequent analysis phases?

In the light of these two studies a final question was also addressed: 
- Do these observations have methodological, analytical and interpretative implications and hence relevance for sampling, storage and examination protocols?

\section{Experiment 1: The reincorporation of Silt and clay-sized particulates on clothing}

\subsection{Methodology}

Ultra-violet powder (median diameter of 15 microns) has been shown to be an accurate proxy for silt and clay-sized soil materials [7, 12] and has the advantage of being able to be identified under ultra-violet light and then quantified at various resolutions using binocular microscopy to establish spatial trends and patterns [12]. In this study the experiment was repeated three times under the same conditions using new, identical and freshly washed long sleeved t-shirts (manufactured by Cedarwood State, 95\% cotton, 3\% polyester) and denim jeans (manufactured by Red Herring, 100\% cotton). Control samples were tested for ultraviolet reflectance and found to be negative. In order to investigate the degree of reincorporation and redistribution that occurred and the spatial pattern of such transfers (whether it varies vertically and whether particular panels or areas are prone to the collection of decayed material) garments were divided into sample areas as shown in Figure 1.

To apply the fluorescent powder to the clothing in a manner that mimicked natural transfer, a standardised procedure was established which involved lightly dusting a shaving brush with the powder and gently flicking the bristles with the index finger 15 times. Care was taken to ensure that only a $20 \mathrm{~cm} \times 20 \mathrm{~cm}$ panel on the t-shirt was seeded with the powder (see area $\mathrm{T}$ in Figure 1) and this was achieved by shielding the rest of the clothing with dust sheets. The transfer of the fluorescent particulates to the clothing was undertaken in a separate location to where the remainder of the experiment took place in order to avoid any effects of background contamination (see, for example, Petterd et al [15]). The subject subsequently engaged in standardised activity for a four hour period (four hours was deemed adequate as Bull et al [7] demonstrate that more than $50 \%$ of powder can be expected to decay from the host surface during this period). The subject remained seated and only stood for short periods (moving to and from a chair).

After four hours, the garments were inspected for the presence of fluorescent powder. Firstly, garments were visually inspected using an ultra-violet lamp in a black-out room. Presence of fluorescent powder and any observations regarding particular patterns or noteworthy features were recorded and subsequently documented using the macro-zoom feature on a digital camera. Secondly, the designated areas were sampled using aluminium SEM sample stubs coated with black carbon discs (to render any powder visible) and were examined using a light microscope lit by an ultra-violet lamp.

\subsection{Results and observations}

In all, thirteen areas of the jeans were sampled in each experimental run and fluorescent powder was visible to the naked eye in all but one instance (area L7 run 3), however 
fluorescent powder was visible microscopically in every area sampled. The spatial distribution of the powder is presented in Figure 2. In each experimental run it was found that the highest concentrations of powder were found at sites L1-3 (the lap area of the jeans). At these sites, the powder was observed in all three runs to accumulate around areas of stitching and creasing which concurs with the finding of Akulova et al. [16] who document a similar phenomenon with fibre evidence. For the samples that had been collected on SEM stubs, a grading system was established to denote the coverage of the stub with fluorescent powder particles (where 5 indicated complete coverage and 0 indicated no particulates present). The average grade for each panel of the jeans was calculated and is presented graphically in Figure 3. This confirms that the highest concentration of particulates were present in the lap area (L1-5) in contrast to the lower part of the t-shirt (U1-4) whilst in comparison there was much lower concentrations of powder on the lower part of the jeans (L6-9).

\section{Experiment 2: The redistribution of pollen particulates on clothing}

\subsection{Methodology}

In the light of the findings of Experiment 1, Experiment 2 was designed to establish the nature of natural transfer of geoforensic trace evidence onto clothing and the subsequent potential for redistribution of that trace evidence during the course of the clothing being seized and packaged for transit to the forensic laboratory for analysis. Pollen was considered to be a suitable form of trace evidence for this experiment as it has been shown to exhibit similar transfer and decay behaviour to other geoforensic materials and is therefore a suitable proxy for a number of types of geoforensic evidence evidence [7]. It also enabled a scenario for the transfer of the pollen onto the clothing to be carried out which mimicked reality as closely as possible [17] by means of a subject wearing brand new and washed jeans walking closely past a row of daffodil (Narcissus $s p$ ) plants. This was repeated three times with new but identical pairs of jeans. The legs of the jeans were divided into 4 equal sections as Figure 4 shows and prior to the experiment being undertaken control samples were taken from each section (all found to be negative for daffodil pollen). After the subject had walked past the daffodils (and brushing past some of the flowers to simulate accidental contact) the subject walked to the laboratory where the jeans were removed and samples taken (using SEM stubs covered with double-sided tape) from the top section of the jeans (A and B in Figure 4).

Each pair of jeans was then folded in the same manner and placed in separate labelled sample bags which were then sealed and left over night. The next day, each of the 4 sections (themselves subdivided into two sections) of the jeans were taped and then using SEM, the number of pollen grains present on each section was counted.

\subsection{Results and Observations}

The numbers of daffodil pollen grains found on each section of each pair of jeans at the different stages of the experiment are presented in Table 1, the average numbers of pollen 
grains found in the main 4 sections after they had been folded and stored is presented in Table 2.

The amount of pollen transferred onto the jeans varied for each run, with run 2 exhibiting a much lower degree of transfer than runs 1 and 3. It was also identified that the amount of pollen transferred to the upper parts of the jeans (A and B) prior to folding varied with no pollen being transferred in run 1, 57 grains in run 2 and 142 grains in run 3. The spatial pattern of pollen on the jeans after folding and packaging was similar for each of the three runs. Secondary transfer from the lower parts of the jeans to the upper parts was observed and resulted in a relatively even spread of the pollen particulates on upper and lower parts of the jeans (see Figure 5). This demonstrates that the spatial distribution of pollen particulates on jeans can be altered by the action of folding and packaging the garment in a manner generally employed for securing and transferring garments to a laboratory for forensic analysis.

\section{Discussion}

Experiment 1 demonstrated that the reincorporation of trace proxy geoforensic particulates occurs both within the garment in question and also to other garments thereby affecting the spatial distribution of trace evidence on clothing. In order to ensure the recovery of as much of the trace particulate evidence as possible it is important to not only sample all areas (upper and lower) of the 'host' garment, but also any lower garments that may have been worn with that 'host' garment. In particular the stitching and creasing details of any garments should be sampled carefully as these were shown to be good retainers of trace evidence.

A significant implication of these findings is the bearing that they have on the interpretation of trace geoforensic evidence that is recovered from clothing. There are many instances where the spatial distribution of trace evidence on a garment has had bearing on the eventual crime reconstruction presented to a court. This study demonstrates that particulates will experience decay but that that decay does not necessarily mean the particulates are lost from the system. Therefore, reincorporation of particulates on the same garment or indeed other garments that are being worn (or are subsequently worn) is likely to have an effect on the decay curve of trace particulates. It is important that this is taken into account when interpreting the trace evidence that is recovered from a garment and establishing a chronology and temporality of pre-, syn- and post-crime events. For example, if a suspect was apprehended twenty-four hours after an event and a significant amount of trace evidence was recovered, it might be interpreted that the material recovered must be from a more recent transfer (Bull et al [7] demonstrate that only small quantities of such material persists after twenty-four hours). However, much of the material recovered may be that which has followed the expected pattern of decay, yet instead of leaving the system, it has been reincorporated at lower levels. Indeed, if a pair of denim jeans were recovered during an investigation and subsequently inspected for the presence of trace sediments, it may well be a complex matter to establish the point of initial contact. For example, if high concentrations 
of particles are located on the upper sections of the jeans then it may be deduced that initial contact took place here when, in fact, material may have been shed from the upper garments which may be the true location of the initial transfer.

Experiment 2 showed that secondary transfer of daffodil pollen particulates occurs when garments are folded and bagged for subsequent analysis which changes the spatial distribution of particulates on that garment. The spatial pattern of particulates on clothing is a potentially vital source of information that may have great bearing on the interpretation of trace particulates which are recovered. For example whether the trace evidence being investigated is present on the front or back of a skirt, or indeed in the upper or lower parts of pair of jeans will provide helpful information as to the possible sources and time frame of the original transfers of evidence onto those garments. Such intelligence is frequently very important in establishing time frames and potential places of contact between victims and suspects or locations and suspects. If this attribute of the trace evidence is deemed to be potentially important in a case, the packaging of garments must be carried out with great care in order to prevent such secondary transfers occurring.

\section{Conclusions}

These two experimental studies have demonstrated that:

- Reincorporation of trace particulates occurs from upper to lower parts of the same garment and also from upper garments to lower garments. Thus the decay of particulates after a contact has been made does not necessarily involve a loss of those particulates from the entire system but rather a redistribution.

- For this scenario whether the subject sat down after the transfer had been made (to simulate driving away from the scene of a crime), reincorporation occurred to all areas of the lower garments, however the highest concentration of particulates was found to be the lap area of the jeans. Particulates also tended to be preserved around technical details such as stitching or relief design features of the garments.

- Folding and packaging items of clothing elicits a redistribution of pollen particulates (and by inference any trace particulate evidence) that are present.

Whilst these are relatively small scale studies, they do demonstrate the importance of an appreciation for the potential for the reincorporation and redistribution of trace evidence for meaningful and accurate interpretations of trace physical evidence on clothing to be achieved. In particular, these findings must be taken into account when reaching conclusions regarding the source of initial contacts (as Chewning et al. [14] demonstrate for fibres) or the chronology and timescales of events [18]. Whilst 'every contact leaves a trace' [19] it is important to never underplay the complexity of those contacts and the preservation of trace evidence over long time frames only adds to these complexities. Sensitive collection 
procedures must be employed so that meaningful analysis is possible that will enable the most coherent and balanced interpretations of that evidence to be reached.

\section{References}

[1] Kirk, P. (1953) Crime Investigation New York: Interscience Publishers

[2] Wiggins, K., and Houck, M. (2001) "Introduction" in Houck, M., ed. Mute Witnesses: Trace evidence analysis London: Academic Press pp.xi-xxxi

[3] Pounds, C., and Smalldon, K. (1975a) "The transfer of fibres between clothing materials during simulated contacts and their persistence during wear: Part I - fibre transference" Journal of the Forensic Science Society 15 17-27

[4] Pounds, C., and Smalldon, K. (1975b) "The transfer of fibres between clothing materials during simulated contacts and their persistence during wear: Part II" Journal of the Forensic Science Society 15 29-37

[5] Pounds, C., and Smalldon, K. (1975c) "The transfer of fibres between clothing materials during simulated contacts and their persistence during wear: Part III - a preliminary investigation of the mechanisms involved" Journal of the Forensic Science Society $15197-$ 207

[6] Hicks, T., Vanina, R., and Margot, P. (1996) "Transfer and persistence of glass fragments on garments" Science and Justice 26 101-107

[7] Bull, P. A., Morgan, R. M., Sagovsky, R., and Hughes, G. (2006) "The transfer and persistence of trace particulates: Experimental studies using clothing fabrics" Science and Justice 46(3) 185-195

[8] Houck, M., ed. (2001) Mute witnesses: Trace evidence analysis London: Academic Press

[9] Robertson, J., and Roux, C. (2000) “Transfer and persistence” In Siegel, J., Saukko, P., and Knupfer, G., eds. Encyclopaedia of Forensic Sciences Academic Press pp.834-838

[10] Morgan, R. M., and Bull, P. A. (2007) "The philosophy, nature and practice of forensic sediment analysis" Progress in Physical Geography 31(1) 43-58

[11] Morgan, R. M., and Bull, P. A. (2007) "Forensic geoscience and crime detection: identification, interpretation and presentation in forensic geoscience" Minerva Medicolegale 127 73-89

[12] Morgan, R. M., Freudiger-Bonzon, J., Nichols, K. H., Jellis, T., Dunkerley, S., Zelazowski, P. and Bull, P. A. (2009) "The forensic analysis of sediments recovered from footwear" In Ritz, K., et al Criminal and Environmental Soil Forensics Netherlands: Springer 
[13] Allen, T. J., Hoefler, K. and Rose, S. J. 1998b The transfer of glass - part 3: the transfer of glass from a contaminated person to another uncontaminated person during a ride in a car. Forensic Science International 93: 195-200

[14] Chewning, D., Deaver, K., and Christensen, A. (2008) "Persistence of fibers on ski masks during transit and processing" Forensic Science Communications 10 (3)

[15] Petterd, C., et al (1999) "Glass particles in the clothing of members of the public in south-eastern Australia - a survey” Forensic Science International 103 193-198

[16] Akulova, V., Vasiliauskiené, D., and Talaliené, D. (2002) "Further insights into the persistence of transferred fibres onto outdoor clothes" Science and Justice 42 165-171

[17] Morgan, R. M., Cohen, J., McGookin, I., Murly-Gotto, J., O’Connor, R., Muress, S., Freudiger-Bonzon, J. and Bull, P. A. (2009) "The relevance of the evolution of experimental studies for the interpretation of some trace physical evidence" Science and Justice 49277 285

[18] Margot, P. (2000) “A question of time” Science and Justice 40(2) 64-71

[19] Locard, E. (1930) "The analyses of dust traces parts I, II and III" American Journal of Police Science 


\section{List of Figures and Tables}

Figure 1 The study sample areas where ' $T$ ' (torso) denotes the $20 \mathrm{~cm} \times 20 \mathrm{~cm}$ seeded panel, ' $\mathrm{U}$ ' indicates the upper trouser and ' $\mathrm{L}$ ' indicates the lower trouser.

Figure 2 The extend of the fluorescent powder redistribution to lower garments from panel ' $\mathrm{T}$ '

Figure 3a The density (average grading) of powder particulates present on each area of the upper and lower garments

Figure $3 \mathrm{~b}$ Table presenting the scores for each samples site

Figure 4 Sample locations on jeans in experiment 2 (not to scale)

Figure 5 Spatial distribution of pollen on each pair of jeans after folding and packaging (for locations A-D see figure 4).

Table 1 The number of pollen grains recovered at each sample point

Table 2 The numbers of grains recovered in each main section after folding and packaging 


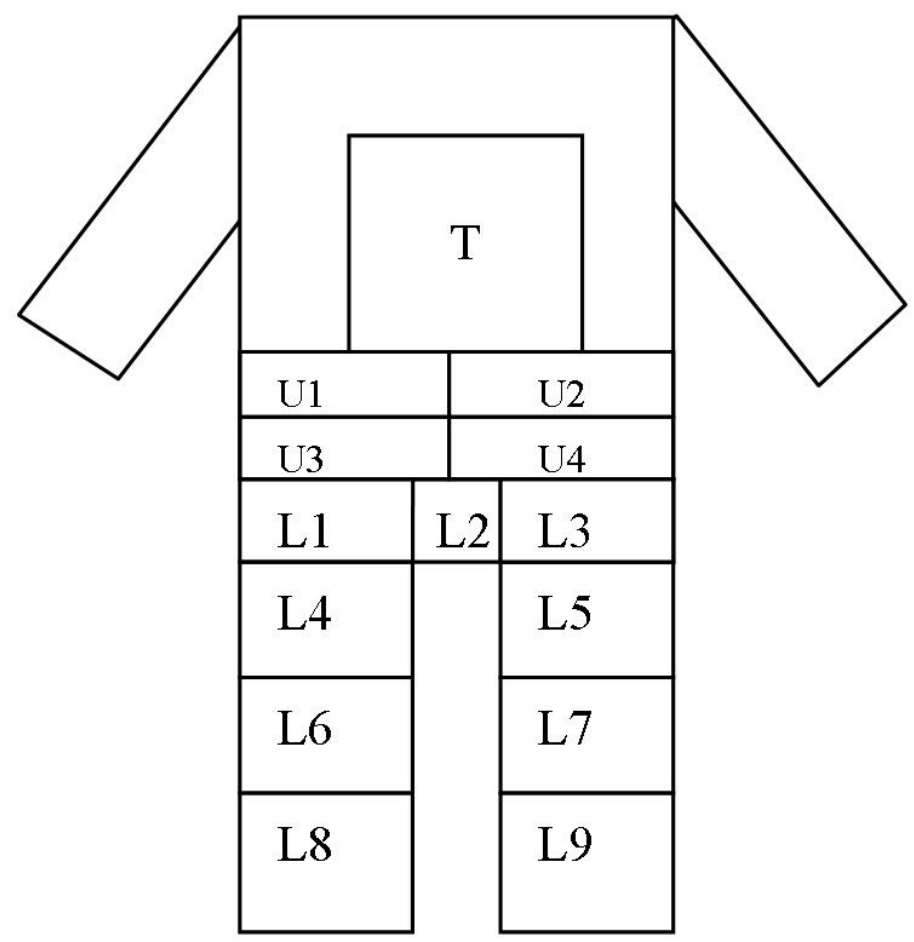

Figure 1 The study sample areas where ' $\mathrm{T}$ ' (torso) denotes the $20 \mathrm{~cm}$ x $20 \mathrm{~cm}$ seeded panel, ' $U$ ' indicates the upper trouser and ' $L$ ' indicates the lower trouser.

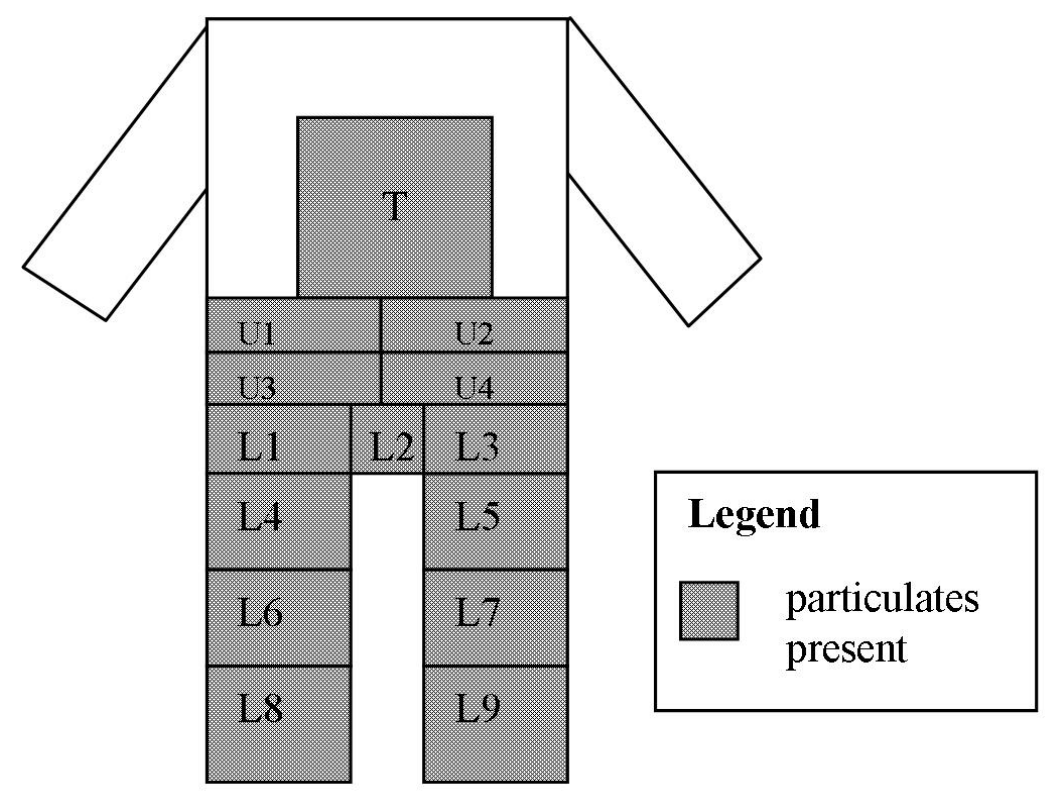

Figure 2 The extent of the fluorescent powder redistribution to lower garments from panel ' $\mathrm{T}$ ' 


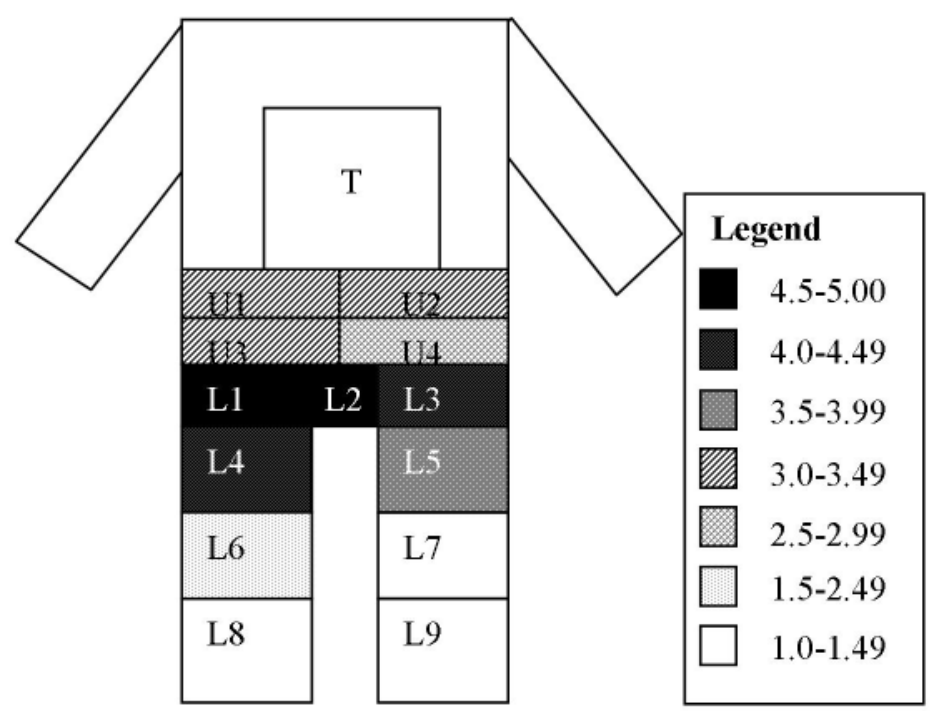

Figure 3a The density (average grading) of powder particulates present on each area of the upper and lower garments

\begin{tabular}{|l|r|r|r|r|}
\hline Sample site & \multicolumn{1}{|c|}{$\mathbf{1}$} & \multicolumn{1}{|c|}{$\mathbf{3}$} & \multicolumn{1}{l|}{ Average } \\
\hline U1 & 3 & 4 & 3 & 3.3 \\
\hline U2 & 3 & 3 & 3 & 3.0 \\
\hline U3 & 2 & 4 & 3 & 3.0 \\
\hline U4 & 2 & 4 & 2 & 2.7 \\
\hline L1 & 5 & 5 & 5 & 5.0 \\
\hline L2 & 5 & 4 & 4.5 & 4.5 \\
\hline L3 & 3 & 5 & 5 & 4.3 \\
\hline L4 & 4 & 4 & 5 & 4.3 \\
\hline L5 & 2 & 5 & 4 & 3.7 \\
\hline L6 & 1.5 & 2 & 1 & 1.5 \\
\hline L7 & 1 & 1 & 1 & 1.0 \\
\hline L8 & 1 & 2 & 1 & 1.3 \\
\hline L9 & 1 & 2 & 1 & 1.3 \\
\hline
\end{tabular}

Figure $3 b$ Table presenting the scores for each samples site 


\begin{tabular}{|l|l|l|}
\hline \multicolumn{2}{|l|}{} \\
\hline $\mathrm{A}_{1}$ & & $\mathrm{~B}_{1}$ \\
\hline $\mathrm{A}_{2}$ & & $\mathrm{~B}_{2}$ \\
\hline $\mathrm{C}_{1}$ & & $\mathrm{D}_{1}$ \\
\hline $\mathrm{C}_{2}$ & \\
\hline
\end{tabular}

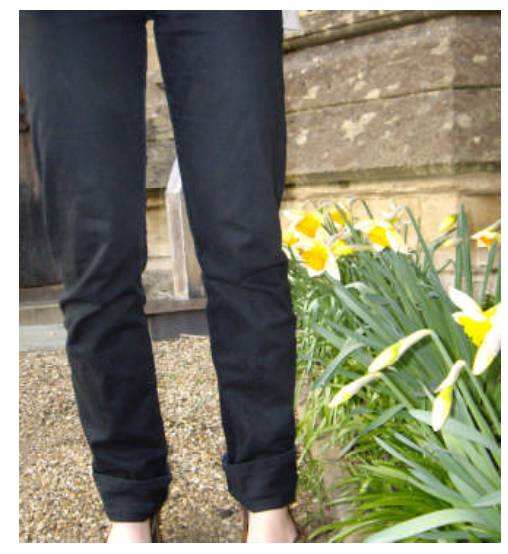

Figure 4 Sample locations on jeans in experiment 2 (not to scale)

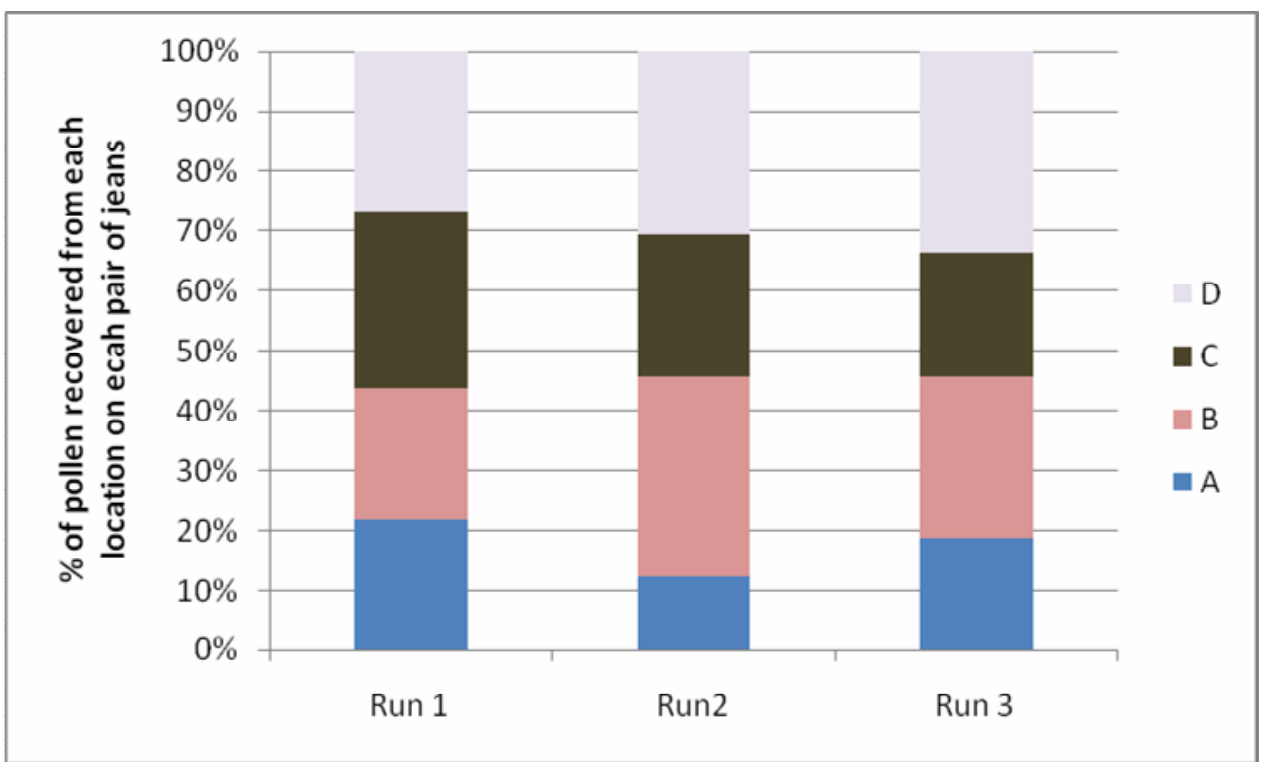

Figure 5 Spatial distribution of pollen on each pair of jeans after folding and packaging (for locations A-D see figure 4). 
Table 1: the number of pollen grains recovered at each sample point

\begin{tabular}{|c|c|c|c|}
\hline $\begin{array}{l}\text { Experimental } \\
\text { Run }\end{array}$ & Stage of experiment & Sample location & $\begin{array}{l}\text { Number } \\
\text { of pollen } \\
\text { grains }\end{array}$ \\
\hline \multirow[t]{10}{*}{ Run 1} & \multirow[t]{2}{*}{ Post forensic event } & $\mathrm{A}$ & 0 \\
\hline & & B & 0 \\
\hline & \multirow[t]{8}{*}{ Post folding and packaging } & $\mathrm{A}_{1}$ (upper left) & 2186 \\
\hline & & $\mathrm{A}_{2}$ (upper left) & 554 \\
\hline & & $\mathrm{B}_{1}$ (upper right) & 1746 \\
\hline & & $\mathrm{B}_{2}$ (upper right) & 1036 \\
\hline & & $\mathrm{C}_{1}$ (lower left) & 951 \\
\hline & & $\mathrm{C}_{2}$ (lower right) & 2739 \\
\hline & & $\mathrm{D}_{1}$ (lower left) & 2512 \\
\hline & & $\mathrm{D}_{2}$ (lower right) & 852 \\
\hline \multirow[t]{10}{*}{ Run 2} & \multirow[t]{2}{*}{ Post forensic event } & $\mathrm{A}$ & 57 \\
\hline & & B & 0 \\
\hline & \multirow[t]{8}{*}{ Post folding and packaging } & $\mathrm{A}_{1}$ (upper left) & 85 \\
\hline & & $\mathrm{A}_{2}$ (upper left) & 213 \\
\hline & & $\mathrm{B}_{1}$ (upper right) & 142 \\
\hline & & $\mathrm{B}_{2}$ (upper right) & 667 \\
\hline & & $\mathrm{C}_{1}$ (lower left) & 213 \\
\hline & & $\mathrm{C}_{2}$ (lower right) & 355 \\
\hline & & $\mathrm{D}_{1}$ (lower left) & 170 \\
\hline & & $\mathrm{D}_{2}$ (lower right) & 568 \\
\hline \multirow[t]{7}{*}{ Run 3} & \multirow[t]{2}{*}{ Post forensic event } & $\mathrm{A}$ & 99 \\
\hline & & $\mathrm{B}$ & 43 \\
\hline & \multirow[t]{5}{*}{ Post folding and packaging } & $\mathrm{A}_{1}$ (upper left) & 880 \\
\hline & & $\mathrm{A}_{2}$ (upper left) & 1405 \\
\hline & & $\mathrm{B}_{1}$ (upper right) & 695 \\
\hline & & $\mathrm{B}_{2}$ (upper right) & 2611 \\
\hline & & $\mathrm{C}_{1}$ (lower left) & 2214 \\
\hline
\end{tabular}




\begin{tabular}{|l|l|l|l|}
\hline \multirow{2}{*}{} & \multirow{2}{*}{} & $\mathrm{C}_{2}$ (lower right) & 298 \\
\cline { 3 - 4 } & $\mathrm{D}_{1}$ (lower left) & 3420 \\
\cline { 3 - 4 } & $\mathrm{D}_{2}$ (lower right) & 681 \\
\hline
\end{tabular}

Table 2 the numbers of grains recovered in each main section after folding and packaging

\begin{tabular}{|c|c|c|c|c|c|}
\hline & \multicolumn{3}{|c|}{ Upper Leg } & \multicolumn{2}{c|}{ Lower Leg } \\
\hline & A & B & C & D & Total \\
\hline Run 1 & 2739 & 2782 & 3690 & 3364 & 12575 \\
\hline Run 2 & 298 & 809 & 568 & 738 & 2413 \\
\hline Run 3 & 2285 & 3307 & 2512 & 4102 & 12206 \\
\hline
\end{tabular}

